\title{
Sphere-and-Point Incidence Relations in High Dimensions with Applications to Unit Distances and Furthest-Neighbor Pairs
}

\author{
F. R. K. Chung \\ Bell Communications Research, Morristown, NJ 07960, USA
}

\begin{abstract}
For $n$ points in three-dimensional Euclidean space, the number of unit distances is shown to be no more than $\mathrm{cn}^{8 / 5}$. Also, we prove that the number of furthest-neighbor pairs for $n$ points in 3-space is no more than $\mathrm{c} n^{85}$, provided no three points are collinear. Both these results follow from the following incidence relation of spheres and points in 3-space. Namely, the number of incidences between $n$ points and $t$ spheres is at most $c n^{4 / 5} t^{4 / 5}$ if no three points are collinear and $n^{3 / 2}>1>n^{1 / 4}$. The proof is based on a point-and-line incidence relation established by Szemerédi and Trotter. Analogous versions for higher dimensions are also given.
\end{abstract}

\section{Introduction}

In [12], Szemerédi and Trotter have established the following result: for $n$ points and $t$ lines in the Euclidean plane with $\sqrt{n} \leq t \leq\left(\begin{array}{l}n \\ 2\end{array}\right)$, the number of incidences between points and lines is no more than $\mathrm{cn}^{2 / 3} t^{2 / 3}$. This result is very useful in dealing with various geometric configurations (see [12]). Efforts have been made to extend this result to higher dimensions involving $n$ points and $t$ spheres. However, the number of such incidences can be as large as $n t$ if the positions of the points and spheres are allowed to be arbitrary. In this paper we obtain the following incidence relation for restricted sets of points and spheres in 3-space.

Theorem 1. For n points and $t$ spheres in 3-space the number of incidences between the points and spheres is at most $c\left(n^{4 / 5} t^{4 / 5}+m^{1 / 2}+n\right)$, provided no three centers of the spheres are collinear.

This follows from Theorem 2 with a relaxed restriction. 
Theorem 2. For $n$ points and t spheres in 3-space, the number of incidences between points and spheres is at most $c\left(n^{4 / 5} t^{4 / 5}+t n^{1 / 2}+n\right)$ if, for any choice of three points, the number of spheres containing the three points is bounded above by some constant $c^{t}$.

There are analogous versions for high-dimensional Euclidean spaces as well as the following version for the plane.

Theorem 3. For n points and circles in the plane, the number of incidences between points and circles is at most $c\left(n^{3 / 4} t^{3 / 4}+n+t\right)$.

The above theorem has been deduced independently by W. T. Trotter and also by Beck (see [3]). For the sake of completeness, we give a proof in Section 2.

Theorem 4. For $n$ points and $t(d-1)$-spheres in d-dimensional Euclidean space, the number of incidences between points and spheres is at most

$$
c\left(n^{(d+1) /(d+2)} t^{(d+1) /(d+2)}+n^{(d-2) /(d-1)}+n\right)
$$

provided no set occurs as an intersection of $i$ spheres in more than $c^{\prime}$ ways where $c^{\prime}$ is a constant (and $c$ depends on $c^{t}$ ), for some $i>1$.

As an immediate consequence of Theorems 3 and 4, we have the following:

Theorem 5. For n points in d-dimensional space, the number of d-spheres containing at least $k$ points is at most

$$
c \frac{n^{d+1}}{k^{a+2}}
$$

if $k>c^{\prime} n^{(d-2) /(d-1)}$ and no set occurs as an intersection of $i$ spheres in more than $c^{\prime}$ ways where $c, c^{\prime}$ are constants (and $c$ depends on $c^{\prime}$ ).

A number of problems can be solved using these theorems, some of which we now describe. We expect this incidence relation will be very useful for many other extremal problems in high-dimensional space as well.

First, we consider the unit distance problem. Let $F_{d}(n)$ denote the maximum possible number of occurrences of a particular distance, say the unit distance, among $n$ points in $d$-dimensional Euclidean space. There is a large literature on the unit distance problem in the Euclidean plane. Erdös [6] first raised this problem in 1946 and he showed that $F_{2}(n)<n^{3 / 2}$. This was subsequently improved by Jozsa and Szemerédi [9], and Beck and Spencer [4]; and the current best upper bound $c n^{4 / 3}$ is due to Spencer et al. [11] in a 1984 paper. The best lower bound currently available for $F_{2}(n)$ is $n^{1+\varepsilon / \log \log n}$ which is obtained by considering the square lattice (see [6]). For three-dimensional space, Erdös [6] proved that

$$
c n^{4 / 3} \log \log n<F_{3}(n)<c n^{5 / 3} .
$$


This was improved by Beck [3] to

$$
F_{3}(n)<n^{13 / 8+o(1)}
$$

We will show:

Theorem 6. In three-dimensional Euclidean space, the number $F_{3}(n)$ of unit distances among $n$ points is no more than $\mathrm{cn}^{8 / 5}$.

Theorem 7. In d-dimensional Euclidean space the number $F_{d}(n)$ of unit distances among $n$ points is no more than $\mathrm{cn}^{2} 2 / d+2$, , provided no three points are in $c^{\prime}$ spheres for some absolute constants $c$ and $c^{\prime}$.

Next, we consider the furthest-neighbor pair problem. For given $n$ points in the plane, two ordered vertices $(u, v)$ are said to be a furthest-neighbor pair if $v$ is a furthest point from $u$. In other words, $d(u, v)=\max _{w} d(u, w)$. The problem of interest is to determine the maximum possible number $G_{d}(n)$ of furthestneighbor pairs for $n$ points in $d$-dimensional space.

It was shown by Avis [1] that $G_{2}(n)=3 n-3$ or $3 n-4$. Edelsbrunner and Skiena [5] proved that $G_{2}(n)=2 n+2\lfloor n / 2\rfloor-3$. For $n$ points in 3-space, $G_{3}(n)$ can be as large as $n^{2} / 4$ as shown in [2]. Let $H_{d}(n)$ denote the maximum possible number of furthest-neighbor pairs for $n$ points in $d$-dimensional space where no three points are collinear. In [5] it was proved that

$$
H_{d}(n) \leq n^{5 / 3} \text {. }
$$

The proof technique is similar to that in [2] where Avis et al. showed that the number of occurrences of the diameter is no more than $c n^{5 / 3}$ among $n$ points positioned in the surface of a convex body in 3-space.

We will prove the following:

Theorem 8. In three-dimensional Euclidean space, the number $H_{3}(n)$ of furthestneighbor pairs among $n$ points is no more than $\mathrm{cn}^{8 / 5}$, provided no three points are in a line.

This can also be generalized to higher dimensions.

Theorem 9. The number $H_{d}(n)$ of furthest-neighbor pairs among $n$ points in $d$-dimensional space is no more than $\mathrm{cn}^{2-2 /(d+2)}$, provided no three points are in a line.

This paper is organized as follows: in Section 2 we establish the incidence relation of circles and points stated in Theorem 3. In Section 3 we prove the incidence relation of spheres and points in 3-space stated in Theorems 1 and 2. In Section 4 we consider higher dimensions and prove Theorems 4 and 5 . In Section 5 we focus on the unit distance problem and prove Theorems 6 and 7 . In Section 6 we deal with the furthest-neighbor pair problem and we show Theorems 8 and 9 . Section 7 contains concluding remarks and related problems. 


\section{An Incidence Relation for Points and Circles}

We start by investigating the number of incidences of circles and points in the plane. Suppose there are $n$ points and $t$ circles. We show that there are at most $c\left(n^{3 / 4} t^{3 / 4}+n+t\right)$ incidences between circles and points and thus prove Theorem 3 .

Now suppose the $i$ th point $v_{1}$ is in $x_{i}$ circles. Suppose the $j$ th circle $C$, contains $y_{j}$ points and let $f\left(v_{1}, C_{j}\right)$ be 1 if $v_{1}$ is in $C_{j}$ and 0 otherwise. We have

$$
\sum_{i} f\left(v_{i}, C_{i}\right)=\sum_{i} x_{i}=\sum_{i} y_{i}=X
$$

We consider the following two possibilities:

Case 1: $t \geq n$. Since we can map all these $x_{1}$ circles to straight lines by an affine mapping which takes $v_{1}$ to infinity, the number of incidences of $n$ points and these $x_{i}$ circles is no more than $c n^{2 / 3} x_{i}^{2 / 3}$ if $x_{i}>n^{1 / 2}$. Otherwise the number of incidences is at most $n+2\left(\begin{array}{l}x_{i} \\ 2\end{array}\right) \leq c n$. (Throughout the paper we use $c$ to denote some appropriate constant.) The total number of such incidences is at most $c\left(n^{2 / 3} x_{i}^{2 / 3}+n\right)$. This is, for fixed $i$,

$$
\sum_{i, k} f\left(v_{i}, C_{j}\right) f\left(v_{k}, C_{i}\right) \leq c\left(n^{2 / 3} x_{i}^{2 / 3}+n\right) .
$$

On the other hand, we have

$$
\sum_{i, j, k} f\left(v_{i}, C_{j}\right) f\left(v_{k}, C_{j}\right)=\sum_{j}\left(\begin{array}{l}
y_{j} \\
2
\end{array}\right)
$$

Therefore

$$
c n X^{2 / 3}+n^{2} \geq c n^{2 / 3} \sum_{i} x_{i}^{2 / 3}+n^{2} \geq \frac{X^{2}}{2 t}-\frac{X}{2} .
$$

This implies $c\left((n t)^{3 / 4}+t\right) \geq X$, provided $t \geq n$.

Case 2: $n \geq t$. We consider, for fixed $i$ and $j$,

$$
\sum_{k} f\left(v_{k}, C_{i}\right) f\left(v_{k}, C_{j}\right) \leq 2
$$

On the other hand, we have

$$
\sum_{i, j, k} f\left(v_{k}, C_{i}\right) f\left(v_{k}, C_{j}\right)=\sum_{i}\left(\begin{array}{l}
x_{i} \\
2
\end{array}\right)
$$


Therefore

$$
2 t^{2} \geq \frac{X^{2}}{2 n}-\frac{X}{2}
$$

This implies $X \leq c\left((n t)^{3 / 4}+n\right)$, provided $t \leq n$. This completes the proof of Theorem 3.

By a stereographic projection of the 2-sphere to the plane, we have the following:

Corollary 1. In a 2-sphere, the number of incidences between $n$ points and 1 circles is at most $c\left(n^{3 / 4} t^{3 / 4}+n+t\right)$.

This result is used to derive the incidence relation between spheres and points of the next section.

\section{Incidence Relation of Spheres and Points in 3-Space}

In this section we only consider points in three-dimensional Euclidean space. We first prove Theorem 1 by showing that the number of incidences between $n$ points and $t$ spheres is no more than $c\left(n^{4 / 5} t^{4 / 5}+t \sqrt{n}+n\right)$, provided no three centers of the spheres are collinear.

Suppose the $i$ th point $v_{r}$ is in $x_{i}$ spheres and the $j$ th sphere $S$, contains $y_{j}$ points. Let $f\left(v_{1}, S_{1}\right)$ be 1 if $v_{1}$ is in $S_{1}$, and 0 otherwise. The total number of incidences is $X=\sum, x_{1}=\sum, y_{1}$. Now we consider, for a fixed $k$,

$$
\sum_{i} \sum_{i} f\left(v_{i}, S_{l}\right) f\left(v_{i}, S_{k}\right)=\sum_{j}\left|S_{j} \cap S_{k}\right| \text {. }
$$

Since no three centers of the spheres are collinear, all the circles $S_{j} \cap S_{k}$ are distinct. The number of incidences of circles and points is exactly $\Sigma_{,}\left|S_{l} \cap S_{k}\right|$. By using Corollary 1 , we have

$$
\sum_{1}\left|S_{j} \cap S_{k}\right| \leq c\left(y_{k}^{3 / 4} t^{3 / 4}+y_{k}+t\right)
$$

On the other hand, we have

$$
\sum_{i, j, k} f\left(v_{i}, S_{j}\right) f\left(v_{1}, S_{k}\right) \geq \sum_{i}\left(\begin{array}{l}
x_{i} \\
2
\end{array}\right)
$$

Therefore, by using Corollary 1, we get

$$
\begin{aligned}
c \sum_{k}\left(y_{k}^{3 / 4} t^{3 / 4}+y_{k}+t\right) & \geq \sum_{i}\left(\begin{array}{c}
x_{i} \\
2
\end{array}\right) \geq \frac{X^{2}}{2 n}-\frac{X}{2}, \\
c\left(t X^{3 / 4}+X+t^{2}\right) & \geq \frac{X^{2}}{2 n}-\frac{X}{2} .
\end{aligned}
$$

This implies $c\left(n^{4 / 5} t^{4 / 5}+t \sqrt{n}+n\right)>X$. This completes the proof of Theorem 1 . 
We note that the noncollinearity is needed only to deduce the fact that the $S_{j} \cap S_{k}$ are all different circles. The same conclusion can be reached if any circle can only be contained in a bounded number of $S$, 's. Therefore Theorem 2 follows immediately.

\section{Incidence Relation of Spheres and Points in Higher Dimensions}

In this section we consider points in $d$-dimensional space with $d \geq 4$. We want to show that for $n$ points and $t$ spheres (or $(d-1)$-spheres to be exact) in $d$-dimensional space, the number of incidences is no more than $c\left(n^{(d+1) /(d+2)} t^{(d+1) /(d+2)}+t n^{(d-2) /(d-1)}+n\right)$, provided some mild restriction on the points is satisfied. The proof is very similar to that of Theorem 1 except we require that, for any $i<d$, the intersection of any $i(d-1)$-spheres is a unique $(d-i)$-sphere (only depending on the choice of the $i$ spheres). Alternately, we can assume that any $(d-i)$-sphere which contains more than $d-i+1$ points can be the intersection of $i(d-1)$-spheres $S$, for a bounded number of choices of $i$ spheres. The statement of Theorem 4 is sufficient to derive the conclusion that the total number of incidences is at most $c\left(n^{(d+1) /(d+2)} t^{(d+1) /(d+2)}+\right.$ $\left.t n^{(d-2) /(d-1)}+n\right)$.

Namely, using the analogous definition in Section 3, we get

$$
\begin{aligned}
c\left(t X^{d /(d+1)}+t^{(d-1) /(d-2)} X^{(d-3) /(d-2)}+X\right) \\
\quad \geq c \sum_{k}\left(y_{k}^{d /(d+1)} t_{y}^{d /(d+1)}+t y_{k}^{(d-3) /(d-2)}+y_{k}\right) \\
\quad \geq \sum_{t, h, k} f\left(v_{1}, S_{j}\right) f\left(v_{r}, S_{k}\right) \\
\quad \geq \sum_{3}\left(\begin{array}{c}
x_{i} \\
2
\end{array}\right) \\
\quad \geq \frac{X^{2}}{2 n}-\frac{X}{2} .
\end{aligned}
$$

By straightforward calculation we get

$$
X \leq c\left(n^{(d+1) /(d+2)} t^{(d+1) /(d+2)}+t n^{(d-2) /(d-1)}+n\right)
$$

and Theorem 4 is proved.

Theorem 5 is an immediate consequence of Theorem 4. Suppose there are $t$ spheres containing $k$ points or more among $n$ points. Then we obtain

$$
k t \leq c\left(n^{(d+1) /(d+2)} t^{(d+1) /(d+2)}+t n^{(d-2) /(d-1)}+n\right) .
$$

By straightforward manipulation we obtain

$$
t \leq c n^{(d+1)} / k^{(d+2)}
$$

provided $k \geq c^{\prime} n^{(d-2) /(d-1)}$. This version is often useful in extremal problems. 


\section{Unit Distances}

In this section we study the problem of unit distances in three and more dimensions. The number of unit distances among $n$ points is exactly the number of incidences between $n$ points and $n$ unit spheres centered at $n$ points. Since any circle can be in at most two of the $n$ unit spheres, we can use Theorem 2 to deduce that there are at most $c n^{8 / 5}$ unit distances. Hence Theorem 6 follows.

For $d \geq 4$, some restrictions are required on the points since there are sets of $n$ points which have $c n^{2}$ unit distances. For example, we can take $n$ points in 4-space with $n / 2$ points of the form $(a, b, 0,0)$ where $a^{2}+b^{2}=\frac{1}{2}$ and other $n / 2$ points of the form $(0,0, c, d)$ with $c^{2}+d^{2}=\frac{1}{2}$. Clearly, there are $\left\lfloor n^{2} / 4\right\rfloor$ unit distances here.

It is not hard to check that the intersection of $d$ distinct unit spheres in $d$-space contains at most two points. However, the condition that any three points are in a bounded number of spheres is needed so that we can then use Theorem 2 to show that there are at most $c n^{22^{2 / d+21}}$ unit distances which proves Theorem 7.

\section{Furthest-Neighbor Pairs}

The number of furthest-neighbor pairs $H_{3}(n)$ among $n$ points in 3-space is exactly the number of incidences of $n$ points and $n$ spheres $S_{t}$ centered at the given points $v_{1}$. To use Theorem 2 to derive $H_{3}(n) \leq c n^{8 / 5}$, we only have to know for any three points, in how many spheres $S_{t}$ they all belong. It is not difficult to see that if three points are in three spheres then the centers of the spheres are collinear. Using the noncollinearity in the assumption of Theorem 8 , the conclusion follows immediately from Theorem 2.

The $d$-dimensional case for $d>3$ follows from the proof of Theorem 2. Namely, for any fixed $i$, the subsets formed by taking intersection of $i$ spheres $S$, are all distinct because no three centers of the spheres are collinear.

\section{Concluding Remarks}

In this paper we discuss upper bounds for the sphere-and-point incidences. It is natural to ask how close these bounds are to the truth. Can we find constructions which will give good lower bounds? The lower bound $n^{4 / 3} \log \log n$ in the unit distance problem in 3-space [6] is, of course, a lower bound for the number of incidences of spheres and points. This lower bound, however, has not been improved for 40 years. If we consider a slight variation, that is the circle-and-point incidences on the 2-spheres, the lower bounds seem to be more difficult to obtain. (We cannot use lattice points any more.) Recently, Erdös and Pach found a lower bound of order $n \log ^{*} n$ for unit distances among $n$ points on the 2-spheres, while the upper bound is $\mathrm{cn}^{4 / 3}$. It would also be of interest to find a superlinear lower bound for the following problem:

Given $n$ points on a 2 -sphere, how many circles can there be with the property that each circle contains four points or more? 
We also remark that on :he problem of furthest-neighbor pairs for $n$ points in 3-space where no three points are collinear, the lower bound for the number $H_{3}(n)$ of furthest-neighbor pairs remains very poor. There is currently no superlinear lower bound for $\mathrm{H}_{3}(n)$.

After the original preparation of this paper, we learned of new improvements in the bounds obtained here, by Edelsbrunner, Guibas, Sharir, and others (private communication); in particular, they obtain a bound of $O\left(n^{3 / 2+\varepsilon}\right)$ for the number of unit distances in 3-space, using a considerably more intricate analysis.

\section{Acknowledgments}

The author would like to thank George Purdy and Tom Trotter for stimulating discussions on this subject.

\section{References}

1. D. Avis, The number of furthest neighbor pairs of a finite planar set. Amer. Math. Monthly 91 (1984), 417-420.

2. D. Avis, P. Erdös, and J. Pach, Repeated distance in space. Preprint, 1986.

3. J. Beck, On the lattice property of the plane and some problems of Dirac, Motzkin, and Erdös in combinatorial geometry. Combinatorica 3 (1983), 281-297.

4. J. Beck and J. Spencer, Unit distances, J. Combin. Theory Ser. A 37 (1984), 231-238.

5. H. Edelsbrunner and S. S. Skiena, On the number of furthest neighbor pairs in a point set. Preprint.

6. P. Erdös, On sets of distances of n points. Amer. Math. Monthly 53 (1946), 248-250. (This paper is also in: P. Erdös, The Art of Counting, MIT Press, Cambridge, MA, 1973).

7. P. Erdös, On sets of distances of $n$ points in Euclidean space. Magyar Tud. Akad. Mat. Kutato Int. Kozl 5 (1960), 165-169.

8. P. Erdös, On some problems of elementary and combinatorial geometry. Ann. Mat. Pura Appl. (IV) 103 (1975), 99-108.

9. S. Jozsa and E. Szemerédi, The number of unit distances in the plane. In Infinite and Finite Sets, 939-950. Colloquia Mathematica Societatis János Bolyai Vol. 10, North-Holland, Amsterdam, 1975.

10. W. Moser and J. Pach, 100 research problems in discrete geometry, 1986.

11. J. Spencer, E. Szemerédi, and W. T. Trotter, Unit distances in the Euclidean plane. Graph Theory and Combinatorics (Proc. Cambridge Conf. on Combinatorics), Academic Press, New York, 1984.

12. E. Szemerédi and W. T. Trotter, Extremal problems in discrete geometry. Combinatorica $3(3-4)$ (1983), 381-392. 